\title{
O papel da Agência Nacional de Vigilância Sanitária na regulação de vacinas
}

\author{
The role of the National Health Surveillance Agency in the regulation of vaccines \\ El papel de la Agencia Nacional de Vigilancia Sanitaria en la regulación de las vacunas
}

Recebido: 31/03/2021 | Revisado: 10/04/2021 | Aceito: 13/05/2021 | Publicado: 01/06/2021

Caroline Severo de Jesus

ORCID: https://orcid.org/0000-0003-4870-4181 Faculdade Guaraí, Brasil

E-mail: carolinesevero2017@gmail.com

Mairy Ferreira Melo Rezende

ORCID: https://orcid.org/0000-0002-0167-8230 Faculdade Guaraí, Brasil

E-mail: mairy_13@live.com

Adriana Keila Dias

ORCID: https://orcid.org/0000-0003-1291-5593 Faculdade Guaraí, Brasil

E-mail: adrianakeiladias@hitmail.com

Giullia Bianca Ferraciolli do Couto

ORCID: https://orcid.org/0000-0002-9768-778X Faculdade Guaraí, Brasil

E-mail: giulliabianca@hotmail.com

Glaucya Wanderley Santos Markus

ORCID: https://orcid.org/0000-0001-8916-1086 Faculdade Guaraí, Brasil

E-mail: glaucyamarkus@outlook.com

Reobbe Aguiar Pereira

ORCID: https://orcid.org/0000-0003-2578-2611

Faculdade Guaraí, Brasil

E-mail: enfreobbe@gmail.com

\section{Resumo}

As vacinas são substâncias utilizadas na prevenção e no controle de doenças, e mesmo sendo eficazes e seguras, estas ainda podem causar eventos adversos que podem ser multifatoriais. Nesse contexto, tem-se aprimorado ao longo dos tempos os meios de regulação no país, surgindo então órgãos como Agência Nacional de Vigilância Sanitária (ANVISA) que por meio de resoluções e normativas realiza a regulação e o registro de vacinas. O presente artigo trata-se de uma revisão bibliográfica narrativa onde utilizam artigos com publicações entre 2014 e 2020 , além de documentos oficiais com valor histórico e legal, com o objetivo de demonstrar o papel da ANVISA no processo de regulação vacinal. Por meio de resoluções e normativas a citada agência realiza a regulação e o registro de vacinas, desempenhando um papel de suma importância na fiscalização do processo de estudos clínicos verificando o cumprimento de todas as normas vigentes, atuando na inspeção de boas prática de fabricação, afim de regularizar a comercialização de vacinas, tendo ainda papel fundamental na própria aplicabilidade dessas vacinas tendo em vista que regulamenta normas mínimas para os locais de vacinação. Com isso, o presente estudo proporciona uma ampla visão acerca do papel da ANVISA no processo de regulamentação de vacinas, contribuindo assim para o conhecimento de suas normativas que visa a segurança da saúde populacional.

Palavras-chave: Regulamentação; Imunização; Diminuição de riscos; Saúde pública.

\begin{abstract}
Vaccines are substances used in the prevention and control of diseases, and even though they are effective and safe, they can cause adverse events that can be multifactorial. In this context, the means of regulation in the country have improved over the years, and bodies such as the National Health Surveillance Agency (ANVISA) have emerged, which, through resolutions and regulations, carry out the regulation and registration of vaccines. This article is a narrative bibliographic review where articles with publications between 2014 and 2020 were used, in addition to official documents with historical and legal value, in order to demonstrate the role of ANVISA in the process of vaccine regulation. Through resolutions and norms, the aforementioned agency carries out the regulation and registration of vaccines, playing an extremely important role in the inspection of the clinical studies process, verifying compliance with all current regulations, acting in the inspection of good manufacturing practices, in order to regularize the commercialization of vaccines, still having a fundamental role in the applicability of these vaccines in view of the regulation of minimum standards for vaccination sites. With this, the present study provides a broad view
\end{abstract}


about the role of ANVISA in the process of regulation of vaccines, thus contributing to the knowledge of its regulations that view the safety of population health.

Keywords: Regulation; Immunization; Risk reduction; Public health.

\section{Resumen}

Las vacunas son sustancias que se utilizan en la prevención y el control de enfermedades y, aunque son eficaces y seguras, pueden provocar efectos adversos que pueden ser multifactoriales. En este contexto, los medios de regulación en el país han mejorado a lo largo de los años y han surgido organismos como la Agencia Nacional de Vigilancia Sanitaria (ANVISA), que a través de resoluciones y reglamentos regulan y registran las vacunas. Este artículo es una revisión bibliográfica narrativa utilizando artículos publicados entre 2014 y 2020, además de documentos oficiales con valor histórico y legal, con el fin de demostrar el papel de ANVISA en el proceso de regulación de vacunas. Mediante resoluciones y normativas, el citado organismo realiza la regulación y registro de vacunas, jugando un papel de suma importancia en la fiscalización del proceso de estudios clínicos, verificando el cumplimiento de toda la normativa vigente, actuando en la fiscalización de buenas prácticas de fabricación, con el fin de regularizar la comercialización de vacunas, teniendo también un papel fundamental en la aplicabilidad de estas vacunas en vista de la regulación de estándares mínimos para los sitios de vacunación. Con ello, el presente estudio aporta una visión amplia sobre el papel de ANVISA en el proceso de regulación de las vacunas, contribuyendo así al conocimiento de sus normativas que apuntan a la seguridad de la salud de la población.

Palabras clave: Regulación; Inmunización; La reducción de riesgos; Salud pública.

\section{Introdução}

As vacinas consistem em substâncias que visam a proteção contra doenças e foi em 1798 que surgiu pela primeira vez esse termo por meio da experiência do inglês Edward Jenner que após ter ouvido rumores de que pessoas que adquiriram varíola bovina não pegavam a varíola, e após introduzir os dois vírus em um garoto observou que esses rumores tinham fundamento científico, assim o nome vacina se derivou do termo Variolae vaccinae nome cientifico da varíola bovina (Brasil, 2016).

No Brasil, desde o século XIX, as vacinas são utilizadas como medida de controle de doenças, elas atuam na prevenção, no controle, e também na eliminação de doenças imunopreveníveis, além disso é bastante custo-efetiva e contribui de forma significativa na redução da morbimortalidade por certos agravos (Brasil, 2014).

Com o passar dos tempos entendeu-se que o cuidado poderia também gerar danos, e mesmo sendo as vacinas um meio seguro de prevenção de doenças, estas ainda podem causar eventos adversos que podem ser multifatoriais (Brasil, 2014 \& Maia, Guilhem, 2016). Para evitar esses eventos é necessário que haja um cuidado especial desde a criação dessas vacinas até o momento de aplicação.

Nesse contexto, tem-se aprimorado ao longo dos tempos os meios de regulação no país, surgindo então órgãos como Agência Nacional de Vigilância Sanitária (ANVISA) que foi criada por meio da lei 9.782, de 1999 e realiza o controle sanitário de produção e comercialização de produtos e serviços inerentes a saúde.

A ANVISA é responsável por coordenar o Sistema Nacional de Vigilância Sanitária (SNVS) e suas ações compreendem aspectos desde a produção até o consumo de bens e serviços inerentes à saúde (Mendes, 2020).

A vacinação é uma importante ferramenta de promoção à saúde, e atualmente em meio a uma pandemia que matou milhares de pessoas, é com uma vacina eficaz que se deposita a esperança de evitar mais mortes.

Com isso é fundamental a atuação das agências reguladoras como a ANVISA que por meio de resoluções e normativas realiza a regulação e o registro de vacinas.

Visto que as vacinas constituem um mecanismo eficiente e importante para a prevenção de doenças e agravos, a presente pesquisa tem o objetivo de demonstrar o papel da ANVISA no processo de regulação vacinal. 


\section{Material e Métodos}

O presente estudo constitui-se em uma revisão bibliográfica narrativa, com abordagem qualitativa, realizada mediante busca em diferentes bancos de dados eletrônicos, abordando o tema Agência Nacional de Vigilância Sanitária na regulação de vacinas. Para tal fim foram consultadas as bases de dados: SciELO, PubMed, Lilacs, Google Acadêmico e Site Oficial da ANVISA. Nas buscas foram utilizados os descritores: ANVISA, Vigilância Sanitária, Vacinas e Imunização.

Para Pereira et al., (2018), refere pesquisa qualitativa como ambiente natural com coleta direta de dados e o pesquisador é o principal instrumento.

Como critérios de inclusão utilizaram-se artigos científicos completos, em português intrínsecos ao tema com publicações entre 2014 e 2020, além de documentos oficiais com valor histórico e legal. Foram excluídas as publicações científicas que não estavam de acordo com a abordagem proposta.

\section{Resultados e Discussão}

A Agência Nacional de Vigilância Sanitária desempenha diversas funções dentre as quais pode ser citado a sua influência na indústria farmacêutica onde vem garantido a segurança das pessoas por meio de fiscalizações qualificadas embasada na preocupação (Stávale, Leal \& Freire, 2020).

Tal agência tem ainda um papel de suma importância na regulação de vacinas, com legislações que visam o controle e segurança dos diversos processos envolvidos desde sua pesquisa, fabricação, regulamentação e até mesmo sua aplicação.

Tratando-se de pesquisa clínica a ANVISA publicou a RDC nº 219/2004 onde se fazia necessário documentos mais detalhados para a solicitação de pesquisa clínica onde a agência verificava do grau de aderência às boas práticas clínicas e à legislação brasileira vigente.

Em 2008 a ANVISA publicou a RDC n 39, fazendo com que sua regulamentação se aproxima-se dos modelos internacionais, permitindo que fosse feita de maneira simplificada a análise de estudos já aprovados em países com agências regulatórias de referência.

Atualmente no Brasil está em vigor a $\operatorname{RDC} n^{\circ}$ 9, de 20 de fevereiro de 2015, que dispõe sobre o regulamento para a realização de ensaios clínicos com medicamentos onde está incluída a submissão do Dossiê de Desenvolvimento Clínico de Medicamento (DDCM) que é composto do conjunto de documentos e informações sobre todas as etapas de desenvolvimento do produto.

A presente RDC foi enriquecida com a Instrução Normativa n 20/2017, que trata dos procedimentos de inspeção em Boas Práticas Clínicas afim de assegurar padrão unificado de eficácia e segurança sanitárias, devendo ser realizada por servidores do quadro efetivo da ANVISA.

A fiscalização das etapas dos estudos clínicos é de extrema importância para que se mantenha a integridade e confiabilidade da pesquisa, mantendo sempre os preceitos da ética.

Outro marco importante para a regulamentação de vacinas foi em 2001 quando a ANVISA publicou a RDC ${ }^{\circ}$ 134/2001, destinada às Boas Práticas de Fabricação (BPF) de medicamentos, estabelecendo um roteiro de inspeção, que foi revogada com a publicação da RDC n ${ }^{\circ} 210 / 2003$, onde instituiu a classificação e os critérios de avaliação de inspeção com base no potencial de risco do processo produtivo. Posteriormente entrou em vigor a RDC n 17/2010, que estabeleceu a validação de sistemas computadorizados de investigação de resultados e foram incluídos itens para água como uso farmacêutico.

Essa nova resolução levou à paralisação de laboratórios públicos, devido à restrição para o compartilhamento de áreas e quanto ao grau das áreas limpas utilizadas para a fabricação dos medicamentos visto que muitos desses laboratórios não tinham instalações apropriadas (Stávale, Leal \& Freire, 2020). 
Com a paralização desses laboratórios houve perdas para a população, visto que diminuiu o número de vacinas disponíveis na rede pública, no entanto um dano necessário para o melhoramento de todo o processo vacinal.

Vigora atualmente a RDC n 301/2019, que segue o Pharmaceutical Inspection Co-operation Scheme (PIC/S) tendo o objetivo de adotar as diretrizes gerais de Boas Práticas de Fabricação de Medicamentos e ampliou o rigor aos elementos que compõem o sistema da qualidade farmacêutico e a necessidade do envolvimento e comprometimento da alta gestão, tendo uma maior abrangência, pois também traz normas quanto aos medicamentos experimentais por meio da Instrução Normativa ${ }^{\circ} 45$.

A ANVISA publicou em 2002 por meio da RDC n 80/2002, a primeira regulamentação de produtos biológicos que exigia a apresentação de informações inerentes à produção e controle de qualidade. Já em 2005 a RDC n 315/2005, apresentava-se mais rigorosa buscando informações sobre a descrição da etapa do processo de fabricação, validação da cadeia de transporte e estudos clínicos.

As resoluções anteriores foram revogadas com a publicação da $\mathrm{RDC} \mathrm{n}^{\circ} 55 / 2010$, que trouxe a necessidade de estudos de imunogenicidade, plano de farmacovigilância e de minimização de riscos e trouxe também recomendações especificas para cada tipo de medicamento biológico, tornando essa RDC um grande avanço para a regulamentação de medicamentos, bem como a RDC n 49/2011, que definiu as alterações pós-registro.

A inclusão de um plano de farmacologia e minimização de riscos consistiu em um ganho enorme para o processo de saúde da população, visto que diminuem a incidência de erros e possíveis agravos.

Em meio à pandemia que se vivência atualmente, a ANVISA publicou a resolução RDC no 348, de 17 de março de 2020, que dispõe sobre os critérios e os procedimentos extraordinários e temporários acerca do registro de medicamentos, produtos biológicos e produtos para diagnóstico in vitro e mudança pós-registro de medicamentos e produtos biológicos, onde será seguida a presente resolução quando ficar configurada a indicação terapêutica específica para prevenção ou tratamento da Covid-19.

A presente resolução detém impacto direto na saúde populacional tendo em mente que a disseminação do novo Coronavírus cresce de maneira aterradora fazendo milhares de vítimas em todo o mundo, sendo fundamental a velocidade e a priorização de ações que visem sua diminuição dos casos e de seus efeitos.

A ANVISA também é a responsável pela RDC n 197, de 26 de dezembro de 2017, que dispõe sobre os requisitos mínimos para o funcionamento dos serviços de vacinação humana que se aplica a todos os serviços que realizam a atividade de vacinação no país, e define normativas quanto aos recursos humanos, condições organizacionais, de infraestrutura, dos registros e notificações das vacinações, dentre outras providências.

A ANVISA exerce regulação direcionado ao consumo, contemplando decisões políticas, detendo seu poder normativo em função da proteção da saúde populacional (Delgado, 2017).

Nesse contexto as normas instituídas por essa agência se fazem fundamentais para toda a população, tendo enfoque na regulação de vacinas, sendo estas um meio seguro e eficaz de prevenir inúmeras doenças.

\section{Considerações Finais}

As vacinas são substâncias essenciais desde sua criação até a atualidade para a prevenção de doenças, sendo uma grande aliada para a saúde populacional.

Em um momento em que se enfrenta uma pandemia que fez milhares de vítimas vê-se a vacinação como uma esperança e como meio de evitar mais mortes e agravos.

Para manter a segurança e garantir a eficácia da vacinação as agências reguladoras desempenham um papel fundamental em nossa sociedade. Nesse contexto a Agência Nacional de Vigilância Sanitária está presente nos diversos processos envolvidos desde a pesquisa, fabricação, regulamentação e até mesmo a aplicação de vacinas. 
Por meio de resoluções e normativas a ANVISA realiza a regulação e o registro de vacinas, desempenhando um papel de suma importância na fiscalização do processo de estudos clínicos verificando o cumprimento de todas as normas vigentes, atuando na inspeção de boas prática de fabricação, afim de regularizar a comercialização de vacinas, tendo ainda papel fundamental na própria aplicabilidade dessas vacinas tendo em vista que regulamenta normas mínimas para os locais de vacinação.

Com isso, o presente estudo proporciona uma ampla visão acerca do papel da ANVISA no processo de regulamentação de vacinas, contribuindo assim para o conhecimento de suas normativas que embasadas em princípios éticos e científicos visão o bem estar e a segurança da saúde populacional.

\section{Referências}

Delgado, J. S. (2017). Limites ao poder regulatório da Agência Nacional de Vigilância Sanitária. 2017. 143 f. Dissertação (Mestrado em Direito) - Instituto CEUB de Pesquisa e Desenvolvimento, Centro Universitário de Brasília, Brasília, 2017.

Maia, C., \& Guilhem, D. (2016). A regulação sanitária brasileira como parte da política de saúde: lacunas e desafios. Rev Panam Salud Publica. 2016;39(5):226-31.

Mendes, G. L. (2020). Ações de vigilância sanitária na área de produtos e serviços farmacêuticos do município de Fortaleza, Ceará. 2020. 104 f. Dissertação (Mestrado em Ciências Farmacêuticas) - Faculdade de Farmácia, Odontologia e Enfermagem, Universidade Federal do Ceará, Fortaleza, 2020.

Ministério da Saúde. Agência Nacional de Vigilância Sanitária. Instrução Normativa n 45, de 21 de agosto de 2019 . Dispõe sobre as boas práticas de fabricação complementares a medicamentos experimentais. Diário Oficial da União 2019; 22 ago.

Ministério da Saúde. Agência Nacional de Vigilância Sanitária. Resolução de Diretoria Colegiada $n^{\circ} 80$, de 18 de março de 2002 . Regulamento técnico de registro, alterações e inclusão pós-registro e revalidação dos produtos biológicos. Diário Oficial da União 2002 ; 19 mar.

Ministério da Saúde. Agência Nacional de Vigilância Sanitária. Resolução de Diretoria Colegiada $n^{\circ} 315$, de 26 de outubro de 2005 . Regulamento técnico de registro, alterações pós-registro e revalidações dos produtos biológicos terminados. Diário Oficial da União 2005 ; 27 out.

Ministério da Saúde. Agência Nacional de Vigilância Sanitária. Resolução de Diretoria Colegiada $n^{\circ} 55$, de 16 de dezembro de 2010 . Dispõe sobre o registro de produtos biológicos novos e produtos biológicos e dá outras providências. Diário Oficial da União $2010 ; 17$ dez.

Ministério da Saúde. Agência Nacional de Vigilância Sanitária. Resolução de Diretoria Colegiada $n^{\circ} 49$, de 20 de setembro de 2011 . Dispõe sobre a realização de alterações e inclusões pós-registro, suspensão e reativação de fabricação e cancelamentos de registro de produtos biológicos e dá outras providências. Diário Oficial da União 2011; 22 set.

Ministério da Saúde. Agência Nacional de Vigilância Sanitária. Resolução de Diretoria Colegiada $n^{\circ} 134$, de 13 de julho de 2001. Determina a todos os estabelecimentos fabricantes de medicamentos, o cumprimento das diretrizes estabelecidas no regulamento técnico das boas práticas para a fabricação de medicamentos. Diário Oficial da União 2001; 14 jul.

Ministério da Saúde. Agência Nacional de Vigilância Sanitária. Resolução de Diretoria Colegiada $n^{\circ} 210$, de 4 de agosto de 2003 . Atualiza as boas práticas de fabricação de medicamentos, com o objetivo de acompanhamento do desenvolvimento de novas tecnologias, nos últimos anos, e a relevância de documentos nacionais e internacionais a respeito do tema. Diário Oficial da União 2003; 14 ago.

Ministério da Saúde. Agência Nacional de Vigilância Sanitária. Resolução de Diretoria Colegiada $n^{\circ} 17$, de 16 de abril de 2010 . Dispõe sobre as boas práticas de fabricação de medicamentos. Diário Oficial da União 2010; 17 abr.

Ministério da Saúde. Agência Nacional de Vigilância Sanitária. Resolução de Diretoria Colegiada $n^{\circ} 301$, de 21 de agosto de 2019 . Dispõe sobre as boas práticas de fabricação de medicamentos. Diário Oficial da União 2019; 22 ago.

Ministério da Saúde. Agência Nacional de Vigilância Sanitária. Resolução de Diretoria Colegiada $n^{\circ} 219$, de 20 de setembro de 2004 . Aprova o regulamento para elaboração de dossiê para a obtenção de comunicado especial (CE) para realização de pesquisa clínica com medicamentos e produtos para a saúde. Diário Oficial da União 2004; 21 set.

Ministério da Saúde. Agência Nacional de Vigilância Sanitária. Resolução de Diretoria Colegiada $n^{\circ} 39$, de 5 de junho de 2008. Aprova o regulamento para a realização de pesquisa clínica e dá outras providências. Diário Oficial da União 2008; 6 jun.

Ministério da Saúde. Agência Nacional de Vigilância Sanitária. Resolução de Diretoria Colegiada $n^{\circ} 9$, de 20 de fevereiro de 2015 . Dispõe sobre o regulamento para a realização de ensaios clínicos com medicamentos no Brasil. Diário Oficial da União $2015 ; 21$ fev.

Ministério da Saúde. Agência Nacional de Vigilância Sanitária. Resolução de Diretoria Colegiada $n^{\circ} 348$, de 17 de março de 2020 . Define os critérios e os procedimentos extraordinários e temporários para tratamento de petições de registro de medicamentos, produtos biológicos e produtos para diagnóstico in vitro e mudança pós-registro de medicamentos e produtos biológicos em virtude da emergência de saúde pública internacional decorrente do novo Coronavírus. Diário Oficial da União 2020; 18 mar.

Ministério da Saúde. Agência Nacional de Vigilância Sanitária. Resolução da Diretoria Colegiada $n^{\circ} 197$, de 26 de dezembro de 2017. Dispõe sobre os requisitos mínimos para o funcionamento dos serviços de vacinação humana. Diário Oficial da União $2017 ; 28$ dez. 
Research, Society and Development, v. 10, n. 6, e35010614512, 2021

(CC BY 4.0) | ISSN 2525-3409 | DOI: http://dx.doi.org/10.33448/rsd-v10i6.14512

Ministério da Saúde. Secretaria de Vigilância em Saúde. Departamento de Vigilância das Doenças Transmissíveis. Manual de Normas e Procedimentos para Vacinação / Ministério da Saúde, Secretaria de Vigilância em Saúde, Departamento de Vigilância das Doenças Transmissíveis. - Brasília: Ministério da Saúde, 2014.

Ministério da Saúde. Vacinas: as origens, a importância e os novos debates sobre seu uso. Instituto de Tecnologia em Imunobiológicos, BioManguinhos/ Fiocruz. 2016.

Pereira A.S. et al. (2018). Metodologia da pesquisa científica. [e-book]. Santa Maria. Ed. UAB/NTE/UFSM. https://repositorio.ufsm.br/bitstream/handle/1/15824/Lic_Computacao_Metodologia-Pesquisa-Cientifica.pdf?sequence=1.

Presidência da República/Casa Civil-Subchefia para Assuntos Jurídicos. Lei $n^{\circ}$ 9.782, de 26 de janeiro de 1999. Define o Sistema Nacional de Vigilância Sanitária, cria a Agência Nacional de Vigilância Sanitária, e dá outras providências.

Stávale, M. C. M., Leal, M. L. F., \& Freire, M. S. (2020). A evolução regulatória e os desafios na perspectiva dos laboratórios públicos produtores de vacinas no Brasil. Cad. Saúde Pública. vol. 36 supl. 2 Rio de Janeiro 2020. 